\title{
La dimensión regional en los planes de ordenamiento territorial del área metropolitana de Bogotá*
}

\section{The Bogotá metropolitan area in the territorial ordering plans' regional dimension}

\author{
Óscar Buitrago Bermúdez** \\ Nohora Inés Carvajal Sánchez ${ }^{* * *}$
}

\section{Resumen}

Se presenta el análisis de la visión regional extraída de los documentos de planeación (Plan de Ordenamiento Territorial -POT-) de Bogotá y algunos municipios de su área de influencia metropolitana. Así mismo, se aporta conocimiento empírico a los interesados en el seguimiento y evaluación de estos planes.

La primer parte del artículo muestra una síntesis del desarrollo histórico del territorio colombiano, con el objeto de señalar que la conformación de las ciudades y áreas metropolitanas ha estado ligada a la transformación histórica del espacio por parte de la sociedad. La segunda presenta algunos indicadores que dan cuenta del crecimiento urbano continuo de Bogotá y de la

\footnotetext{
* Este artículo presenta algunos aspectos de la investigación "Evaluación de la dimensión urbano regional en los planes de ordenamiento territorial del área metropolitana de Bogotá", auspiciada por la Dirección de Investigaciones de la Universidad Pedagógica y Tecnológica de Colombia (UPTC) y adelantada por el grupo de trabajo Visión Territorial de la Maestría en Geografía de la UPTC en convenio con el Instituto Geográfico Agustín Codazzi, en la que los autores participaron como coinvestigadores.

** Ingeniero Agrícola, Magíster en Geografía. Profesor de la Universidad del Valle, Facultad de Humanidades, Departamento de Geografía. Correo e.: osbuber@univalle.edu.co

*** Socióloga, Ingeniera Industrial, Magíster en Geografía. Correo e.: nohoracarvajal@yahoo.com
} 
consolidación del fenómeno de metropolización; además, muestra diferentes modelos de explicación de la jerarquía urbana colombiana, los cuales coinciden en señalar a Bogotá como el principal centro urbano del país que cumple la función de organizador y dinamizador del sistema de ciudades y, a su vez, de la región metropolitana circundante. La tercera parte presenta el análisis de la visión regional de los POT de Bogotá y de una muestra de municipios del área de influencia metropolitana. Para ello se consideraron aspectos como la visión, objetivos, formulación, estrategias e instrumentos y se identificaron ideas concretas acerca de la concepción y proyección de las relaciones entre cada municipio y Bogotá. Finalmente, se concluye que entre el marco de referencia para formular los POT de los municipios mencionados y la visión regional planteada en ellos no existe cohesión espacial ni tempo ral. Por tanto, los procesos de planificación regional en la zona metropolitana de Bogotá cuentan con un gran limitante instrumental.

Palabras clave: Plan de Ordenamiento Territorial, POT, Visión regional, Área metropolitana de Bogotá.

\section{Abstract}

The regional vision's analysis presented, is taken from Bogotá's and some metropolitan influence area municipalities planning's $\sim$ POT $\sim$ documents. It also offers empirical information for those interested in monitoring and evaluating these plans.

The first part is a Colombian territory historic development's synthesis, with the purpose to show that the cities and metropolitan areas' shaping, has been linked to the historical and social space transformation. The second part, presents some indicators that show Bogotás continuous urban growth and the metropolis phenomenon's consolidation. It also presents some explanatory's models of the Colombian urban hierarchy, which coincide in placing Bogotá as the country's main city, that functions as a city system's which organizes and revitalizes itself, and at the same time, the surrounding metropolitan region. The third part, presents an analysis of the Bogotá's POT "regional vision", extracted from the planning documents and from a sample of the metropolitan influence area's municipalities. In order to achieve this, it was necessary to take into account aspects such as: vision, objectives, formulation, strategies and instruments, and also were identified some ideas about the relations between each municipality and Bogotás conception and projection. Finally, the conclusion is that there isn't a spatial neither a temporary cohesion between the municipalities analyzed's reference's framework, created in order to formulate the stated POT and the "regional vision". Therefore, the metropolitan zone of Bogotá regional planning processes have great limitations with working instruments

Key words: Territorial Ordering Plan, POT, Regional vision, Bogotá's Metropolitan area.

22 Óscar Buitrago B. - Nohora I. Carvajal S. 


\section{Introducción}

La visión regional extraída de los documentos de planeación (Plan de Ordenamiento Territorial -POT-) de Bogotá y algunos de los municipios de su área de influencia metropolitana es el eje estructurante de este trabajo, con el cual se busca mostrar el tipo de articulación planteada a nivel regional y aportar conocimiento empírico a los interesados en el seguimiento y evaluación de estos planes.

Para ello se realizó, en primer lugar, una síntesis del desarrollo histórico del territorio colombiano, con el objeto de señalar que la conformación de las ciudades y áreas metropolitanas ha estado ligada a la transformación histórica del espacio por parte de la sociedad. Luego, se indagó por las características que dan cuenta del crecimiento urbano continuo de Bogotá, mediante algunos indicadores de la consolidación del fenómeno de metropolización; este último punto se complementa en la comparación de los diferentes modelos de explicación de la jerarquía urbana en Colombia, que coinciden en señalar a Bogotá como el principal centro urbano del país que cumple la función de organizador y dinamizador del sistema de ciudades y, a su vez, de la región metropolitana circundante. La base teórica construida permite sustentar que tanto las directrices y orientaciones para el ordenamiento territorial del departamento de Cundinamarca, que constituyen el marco de referencia para formular los instrumentos de los POT de los municipios de su jurisdicción, como la visión regional planteada en los diferentes POT, teniendo en cuenta las relaciones horizontales, verticales y temporales entre los diferentes entes territoriales involucrados, no presentan cohesión ni espacial ni temporal, llegándose a concluir que los procesos de planificación regionales en la zona metropolitana de Bogotá cuentan con un gran limitante instrumental, al menos durante los siguientes dos periodos de gobierno.

\section{Desarrollo histórico del territorio colombiano}

El proceso de conformación de las ciudades colombianas ha estado ligado a la transformación histórica del espacio por parte de la sociedad, atendiendo a sus necesidades e intereses. Pero también han contribuido a ese proceso las historias particulares de cada localidad, las historias nacionales que atraviesan los planos locales y la mundialización o proceso que atraviesa los planos nacional y local. Es decir, que en este proceso histórico de construcción de ciudad, los distintos factores económicos, sociales, culturales y políticos son los que le dan contenido.

Según Sarmiento y Castillo (1998), se estima que la ocupación humana del territorio colombiano data de unos veinte a treinta mil años. Hacia finales del siglo xv, en Colombia existían poblados en la región Caribe (culturas 
zenú y tairona), región Andina (muiscas) y sudoeste del país (extensiones del imperio incaico hasta el Valle del Cauca). La población tendía a concentrarse en la zona andina, especialmente en la región cundiboyacense, en el alto y medio Cauca y en el alto y medio Magdalena.

La lógica económica y militar de los españoles contribuyó al cambio de este sistema de poblamiento aborigen, produciendo devastación demográfica (despoblamiento en la Costa Atlántica y disminución de la población andina) y la imposición de un régimen de servidumbre.

Durante el poblamiento español, iniciado a principios del siglo xvI, se establecieron poblados en la costa caribe colombiana, que desaparecieron poco tiempo después. En el interior del país, los núcleos urbanos se emplazaron sobre los antiguos poblados indígenas.

De acuerdo con los mencionados Sarmiento y Castillo (1998), en esta etapa de ocupación española se configuraron cinco grandes unidades espaciales socioeconómicas: 1) Santa Marta y Cartagena, puntos de conexión entre las regiones productoras de metales preciosos y otros bienes de exportación y el exterior; 2) Noroccidente minero, conformado por los actuales departamentos de Antioquia, Caldas, Risaralda y Quindio; 3) Suroccidente minero, agrícola y ganadero, compuesto por los actuales departamentos de Valle del Cauca, Chocó y Cauca; 4) Centrooriente agrícola y ganadero, en donde Bogotá cumple funciones políticas, sociales y comerciales, y Tunja, por su parte, se consolida como un centro de producción y distribución de textiles, y 5) Nororiente artesanal, constituido por Santander: Socorro, principal centro manufacturero del país, y Cúcuta, especializado en la producción de cacao.

Hacia finales del siglo XvIII, el $80 \%$ de la población se concentraba en la región Andina, atendiendo al siguiente orden: Cundinamarca, Boyacá, Cauca, Antioquia y región Atlántica.

Entre 1820 y 1850, según Cuervo y González (1998), se presenta una reorganización socioeconómica del país en torno a la expansión del sector exportador de materias primas. Las principales características de la época son: 1) débiles tasas de crecimiento poblacional, 2) pérdida de población en la región Atlántica por las guerras de independencia y 3) expansión del mestizaje en el interior del país.

En el periodo comprendido entre 1850 y 1870, en distintas áreas del país se concentran bonanzas exportadoras así: 1) Ambalema, Carmen de Bolívar y Palmira: tabaco; 2) Cauca y Antioquia: oro; 3) Valle del Cauca, Fusagasugá, Alto Magdalena, Tolima y Santander: quina.

24 Óscar Buitrago B. - Nohora I. Carvajal S. 
Algunas de las transformaciones socioespaciales más importantes se presentan en la costa Atlántica, en donde Barranquilla toma el liderazgo que tenía Cartagena -debido al comercio internacional y al movimiento de mercancías del interior del país a través del río Magdalena-, y en las zonas cálidas de la región Andina, colonizadas como consecuencia del afán de búsqueda de productos de exportación.

Hacia 1870-1890, el café, concentrado en las tierras templadas de los Andes, especialmente Cundinamarca, Tolima y Santander, se consolida como producto de exportación. Este aspecto influye en el esfuerzo de articulación de las regiones con el mercado externo, lo que conduce a la construcción de líneas férreas, a la iniciación de proyectos para mejorar la conexión entre Cali, Bogotá, Medellín y Barranquilla (que para la época constituían una multipolaridad regional), a la disputa entre Cartagena y Barranquilla por la hegemonía portuaria sobre el Caribe, a la búsqueda de salida al Océano Pacífico por Buenaventura y a la constitución de los ríos Cauca y Magdalena como principales ejes de transporte.

El poblamiento entre finales del siglo XIX y 1930 se caracteriza por el inicio de la industrialización y la urbanización en Colombia, procesos ligados al desarrollo del café y a la transformación de su estructura productiva de hacienda a cultivo campesino, lo cual facilitó el desarrollo de las ciudades al tener que cumplir funciones económicas como el comercio o el beneficio del café. Así mismo, el transporte del grano hizo que fuera prioritaria la constitución de una red de transporte.

Cuervo y González (1998) afirman que hacia 1915 las cuatro principales ciudades del país eran: Medellín, primer centro regional y nacional, especializado en la industria textil; Bogotá, que manejaba las industrias de alimentos, bebidas y calzado; Barranquilla, que también trabajaba la industria textil, y Cali, que se ocupaba de la transformación de la caña de azúcar.

Durante este período se presenta la competencia entre Medellín y Bogotá por la preponderancia nacional, la consolidación de un corredor urbano occidental andino, la creación de diversos polos urbanos como consecuencia de la expansión de la industria del café (Medellín, Manizales, Pereira y Armenia) y la constitución de las cuatro ciudades mayores como centros industriales de una región diferente.

Desde mediados del siglo $\mathrm{xx}$, hasta los años sesenta y setenta, se inicia el proceso de cambio de una sociedad rural a otra urbana. En las doce ciudades más grandes que existían en 1951 (Bogotá, Cali, Medellín, Barranquilla, Bucaramanga, Cartagena, Cúcuta, Manizales, Ibagué, Pereira, Armenia y Palmira) se concentraba el 
$18 \%$ de la población del país; mientras que regiones como la Orinoquia y Amazonia concentraban solamente el 2\% (Sarmiento y Castillo, 1998).

Este periodo se caracteriza por la diversificación industrial (Bogotá: bienes de consumo durable; Medellín y Cali: textiles y productos químicos, y las ciudades intermedias: bienes de consumo intermedio), el crecimiento urbano, la expansión de la agricultura comercial (que estimuló el crecimiento de algunas ciudades como Ibagué, Neiva, Sincelejo y otras del Valle) y la apertura de nuevos frentes de colonización (la Amazonia, el Magdalena Medio, la costa Pacífica y el Catatumbo), que dio importancia a ciudades como Villavicencio y Florencia.

A lo largo del siglo xx se presenta un proceso de primacía urbana colombiana. Según Cuervo y González (1998), la preponderancia de Bogotá y la consolidación de cuatro centros regionales se presentan después de 1920. Se conforman tres grandes corredores urbanos: el andino oriental y occidental, el Atlántico y otro, en formación, constituido por el piedemonte llanero.
Este proceso de primacía urbana se desarrolla con más fuerza en las últimas cuatro décadas, período en el cual Colombia pasó de una estructura cuadricefálica $^{1}$ a otra destacada por el predominio de Bogotá (primera gran metrópoli nacional, mientras que Medellín, Cali y Barranquilla, se constituyen en metrópolis regionales). Según Gouëset (1998), la primacía urbana de Bogotá está relacionada con la participación creciente en la actividad económica del país, la concentración de una tercera parte de la industria nacional, el $40 \%$ del mercado automovilístico, el $50 \%$ de la actividad bancaria y el $60 \%$ del parque informático.

De acuerdo con el estudio reciente (octubre 2003) de la Contraloría de Bogotá: De Bogotá a la región, apuntes para un modelo de desarrollo regional, la ciudad capital cumple múltiples funciones en sus varios radios de influencia.

- El papel internacional está dado por: 1) la concentración de la mayoría de los servicios de telecomunicaciones y de transporte aéreo, 2) tiene la primacía en la producción del país, con una elevada participación en el PIB del

1 Según Gouëset (1998), fue Castells el que propuso el término de macrocefalia urbana, para designar la concentración primacial acelerada que caracteriza a las ciudades de América Latina. De origen médico, supone que la hipertrofia de las metrópolis de América Latina es una patología y que la concentración de actividades y de población en un número reducido de ciudades es excesiva y constituye un obstáculo al desarrollo. Por tanto, la cuadricefalia es la concentración primacial en cuatro ciudades.

26 Óscar Buitrago B. - Nohora I. Carvajal S. 
$21 \%, 3)$ acoge la mayor parte de la inversión extranjera y 4) es el principal centro financiero y bursátil.

- Como única metrópoli nacional, se convierte en la ciudad en la cual tienen asiento los poderes centrales del Estado en sus principales ramas y órganos y concentra la mayor parte de los empleos públicos de alto nivel.

- Su carácter de centro subnacional se evidencia porque existen 296 ciudades y poblaciones cuyas demandas se satisfacen en la ciudad capital.

- El rol de centro regional (o área metropolitana) se determina porque existen más de 19 municipios que reciben una influencia directa de la economía y la actividad bogotana, medida por el flujo de mercancías y personas que se movilizan.

- En su condición de ciudad central, se aprecia en el fenómeno de conurbación que se ha generado en las últimas décadas, según el cual poblaciones cercanas van siendo absorbidas en forma acelerada, incontrolada y periférica.

El proceso de metropolización, característico de finales del siglo $\mathrm{xx}$, es entendido como la intensificación de las relaciones físicas, económicas y sociales entre entidades territoriales vecinas, como resultado del creci-miento de una ciudad, generalmente capital nacional o regional, que actúa como núcleo del fenómeno (Montañez,
1998). En el caso del área metropolitana de Bogotá, los municipios que la conforman son: Soacha, Mosquera, Funza, Cota, Chía, La Calera, Sibaté, Bojacá, Madrid, Tenjo, Tabio, Cajicá, Sopo, Guasca, Facatativá, Suba-choque, Zipaquirá, Tocancipá, Guatavita, Cogua, Nemocón, Gachancipá, Sesquilé, Suesca, Chocontá y Villapinzón.

\section{Crecimiento urbano continuo de Bogotá y conformación de su área metropolitana}

La metrópoli contemporánea está hecha de contrastes: lugar de producción y de reproducción, de creatividad y de oportunidad, de vanguardia y de supervivencia, de abundancia y de hacinamiento, afirma Albet (2000). Bogotá y su área metropolitana reflejan buena parte de estas características, acumuladas en un proceso de largo tiempo.

Así, Bogotá ha experimentado un rápido crecimiento demográfico, debido principalmente a la migración generada por factores de diversas índoles. Entre otros, el despegue industrial de la capital, la violencia política en el campo, la introducción del cambio técnico en la producción agropecuaria en algunas regiones de Colombia, la brecha entre las condiciones de vida en el campo y la ciudad, el fortalecimiento de Bogotá como la capital del país y la persis- 
tencia de un modelo político centralista hasta años recientes (Misión Siglo XXI). Sumado a lo anterior, la ciudad cuenta con una amplia red de comunicaciones y es cercana a zonas de minifundio (Boyacá y Cundinamarca) en continuo proceso de fragmentación y expulsión de población. Jaramillo (1994) presenta claramente el proceso de expansión de Bogotá a partir de su explosivo crecimiento:

- Entre 1930 y 1950 se inicia el mercado de tierras periféricas de Bogotá, en general tierras muy fértiles. Estas se encontraban en manos de terratenientes en proceso de quiebra, hecho que derivó en la apropiación de los terrenos por parte de los capitalistas urbanos, que eran totalmente ajenos a las actividades agropecuarias; estos iniciaron un proceso de parcelación de los terrenos periféricos, dotándolos de cierta infraestructura urbana, previo a su venta.

- Entre 1950 y 1970, la ciudad creció con mayor ritmo que durante el periodo anterior. La suburbanización continuó y favoreció la aparición de una mayor segregación socioespacial, a tal punto que Bogotá se bipolariza: en el norte se desarrollaron los barrios para las clases ricas, en tanto que en el sur se ubicaron los grupos pobres de la sociedad. La parcelación de terrenos en la periferia continuó y se privilegió la construcción en baja densidad con gran consumo de espacio. Los urbanizadores, que en el anterior periodo únicamente parce- laban, en este periodo adicionaron a su actividad la construcción de vivienda. En este periodo pareciera que la cantidad de espacio para el crecimiento de la ciudad fuera ilimitada.

- Entre 1970 y 1988 , el tamaño alcanzado por la ciudad hace que las distancias comiencen a aparecer como obstáculos para la suburbanización; de igual manera, el crecimiento de la ciudad hace que comience a tropezarse con barreras físicas, es decir, que inicia la escasez de terrenos para la construcción. Aparece el desarrollo de áreas no ocupadas y la de densificación de los barrios de clases altas, hechos que propician nuevos espacios en la ciudad para estas clases sociales. La motorización de las clases sociales altas permite la suburbanización de una periferia norte muy extrema. La notoria elevación de los precios de la tierra para este periodo obedece, principalmente, a dos factores: por un lado, a la expansión de la ciudad, y por otro, al deseo de los propietarios de los terrenos adecuados para urbanizar de disputar parte de las ganancias con los promotores urbanizadores, llegándose hasta la especulación.

Actualmente, Bogotá es una ciudad que sigue expandiéndose sobre suelos de alta potencialidad natural, desbordando su propio territorio y promoviendo la urbanización de los cascos urbanos de los municipios aledaños, que a su vez crecen sobre el fértil suelo de la sabana.

28 Óscar Buitrago B. - Nohora I. Carvajal S. 


\section{Indicadores de la metropolización de Bogotá}

El estudio del fenómeno de metropolización de la sabana de Bogotá se ha abordado de diversas maneras. A continuación se presentan algunos indicadores que dan cuenta de ese fenómeno.

4.1 Contigüidad espacial de los municipios vecinos a la capital, conformando anillos metropolitanos: los efectos del crecimiento de Bogotá sobre la sabana se evidencian en un acelerado proceso de urbanización, conurbación y suburbanización, que, en últimas, demuestra la presencia del fenómeno de metropolización en este espacio geográfico. La Misión Bogotá Siglo XXI, en su estudio El Futuro de la Capital (1994), indaga acerca de las relaciones entre Bogotá y su entorno regional, con el objetivo de establecer criterios para la definición de escenarios futuros. Esta investigación parte de definir las diferencias espaciales entre los municipios de la sabana, para lo cual establece inicialmente anillos metropolitanos por contigüidad con la capital, como se observa en la tabla 1 y en el mapa 1.

Tabla 1. Anillos metropolitanos

\begin{tabular}{|l|l|}
\hline ANILLO & MUNICIPIOS QUE LO CONFORMAN \\
\hline Primer anillo & Soacha, Mosquera, Funza, Cota, Chía, La Calera \\
\hline Segundo anillo & Sibaté, Bojacá, Madrid, Tenjo, Tabio, Cajicá, Sopó y Guasca \\
\hline Tercer anillo & Facatativá, Subachoque*, Zipaquirá, Tocancipá y Guatavita \\
\hline Sabana periférica & $\begin{array}{l}\text { Cogua, Nemocón, Gachancipá, Sesquilé, Suesca, Chocontá, } \\
\text { Villapinzón }\end{array}$ \\
\hline
\end{tabular}

* Este municipio fue dividido en dos, dando origen al municipio de El Rosal, que para efectos de este estudio hace parte del tercer anillo.

4.2 Dinámica de la población. En la tabla 2 se muestra el porcentaje de variación de las proyecciones de población entre 2000 y 2005, por anillo metropolitano. Se destaca que el tercer anillo presenta una de las mayores variaciones $(12,7 \%)$, atribuida a que en él se encuentran municipios como Facatativá y Zipaquirá, que cuentan con un alto grado de urbanización debido al efecto de su papel como polos de relevo principal entre Bogotá y los municipios de la vertiente occidental de la cordillera Oriental. La región periférica del suroccidente presenta 


\section{Mapa 1. Anillos metropolitanos de Bogotá}

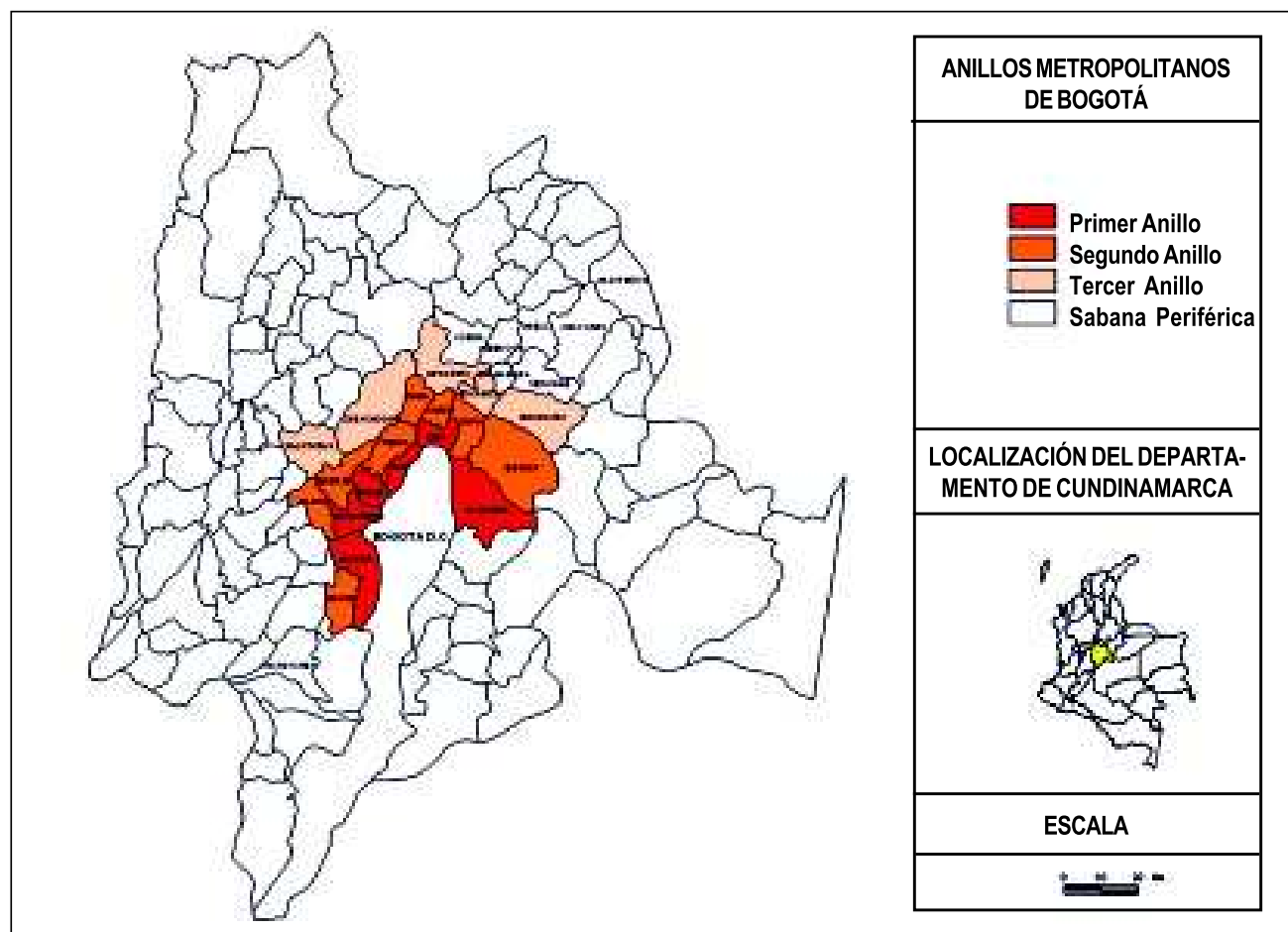

el mayor porcentaje de variación $(13,4 \%)$, hecho que se debe a la presencia del municipio de Fusagasugá, que mantiene una relación de centro de relevo principal con la capital. El primer y el segundo anillos se comportan de manera similar, presentando un porcentaje de variación poblacional impor-tante, mientras que la sabana periférica crece en la mitad de esta proporción.

4.3 Movimiento poblacional, procesos de redensificación y dinámica de los municipios contiguos. Según Dureau (2002), el nuevo modelo de desarrollo metropolitano se caracteriza por ser endógeno (es decir, las movilidades intraurbanas se vuelven el principal factor de la dinámica poblacional de Bogotá), centripeto (el espacio ya urbanizado experimenta transformaciones y procesos de redensificación) y determinado por la dinámica de los municipios contiguos como Soacha y Chía, sobre los cuales se da el extendimiento urbano, o como Madrid, que polariza el proceso de metropolización.

Así, el proceso de expansión de los barrios periféricos populares más allá de los límites del Distrito, como en el caso del municipio Soacha, lo ha

30 Óscar Buitrago B. - Nohora I. Carvajal S. 
Tabla 2. Proyecciones de población por anillo metropolitano

\begin{tabular}{|c|c|c|c|c|}
\hline ANILLO & $\begin{array}{l}\text { Municipios que } \\
\text { lo conforman }\end{array}$ & $\begin{array}{l}\text { Proyecciones } \\
\text { de población } \\
2000\end{array}$ & $\begin{array}{l}\text { Proyecciones } \\
\text { de población } \\
2005\end{array}$ & $\begin{array}{c}\% \\
\text { Variación }\end{array}$ \\
\hline Primer anillo & $\begin{array}{l}\text { Soacha, Mosquera, Funza, } \\
\text { Cota, Chía, La Calera }\end{array}$ & 464.205 & 518.054 & 11,6 \\
\hline Segundo anillo & $\begin{array}{l}\text { Sibaté, Bojacá, Madrid, Tenjo, } \\
\text { Tabio, Cajicá, Sopó y Guasca }\end{array}$ & 185.199 & 206.393 & 11,4 \\
\hline Tercer anillo & $\begin{array}{l}\text { Facatativá, Subachoque, } \\
\text { Zipaquirá, Tocancipá } \\
\text { y Guatavita }\end{array}$ & 216.685 & 244.146 & 12,7 \\
\hline $\begin{array}{l}\text { Sabana } \\
\text { periférica }\end{array}$ & $\begin{array}{l}\text { Cogua, Nemocón, Gachancipá, } \\
\text { Sesquilé, Suesca, Chocontá, } \\
\text { Villapinzón }\end{array}$ & 87.432 & 93.268 & 6,7 \\
\hline $\begin{array}{l}\text { Región } \\
\text { periférica del } \\
\text { Suroccidente }\end{array}$ & Granada, Silvania, Fusagasugá & 138.957 & 157.618 & 13,4 \\
\hline TOTAL & & 1.092 .478 & 1.219 .479 & 11,6 \\
\hline
\end{tabular}

Fuente: Proyecciones de población por área según municipios. DANE. 1999. Variación calculada por los autores a partir de las proyecciones de población del DANE.

convertido en un barrio de Bogotá. Afirma Dureau que el crecimiento acelerado de este municipio no es más que la manifestación del extendimiento de los suburbios de una capital que tiene un ritmo de crecimiento todavía sostenido.

Para el mismo autor, el municipio de Chía, desde hace unos veinte años, viene recibiendo el impacto de las prácticas residenciales de las familias de clase alta de Bogotá, que buscan una mejor calidad de vida en las afueras de la ciudad. La construcción no controlada de urbanizaciones está destruyendo rápidamente el "cinturón verde" indispensable para la población de la metrópoli, peligrando la función de esparcimiento que hasta el momento ofrece este municipio.

La dinámica del municipio de Madrid es distinta, pues la floricultura, al tiempo que limita la expansión de la cabecera municipal, atrae importantes flujos migratorios; de esta manera, mientras la migración hacia Madrid es producto de una atracción propia de las actividades económicas presentes en el municipio, en el caso de Soacha es resultado de la migración hacia Bogotá y su periferia metropolitana. 
4.4 Transporte diario entre Bogotá y los municipios vecinos. El Plan Maestro de Transporte para Bogotá, elaborado en 1995 por la Agencia Japonesa de Cooperación (JICA), muestra, mediante datos estadísticos, los niveles de metropolización entre la capital y los municipios vecinos (Chía, Cajicá, Cota, Tenjo, Funza, Mosquera, Soacha y la localidad de Usme). De acuerdo con este estudio, de los 14,9 millones de viajes que se realizan diariamente en la capital, el $98 \%$ son de sus residentes, lo que indica que solamente el $2 \%$ corresponde a viajes desde o hacia fuera de la ciudad, señalando un bajo grado de metropolización; sin embargo, el porcentaje de vehículos privados que circulan en Bogotá y que están registrados en los municipios vecinos asciende al 33\%.

En cuanto a la fuerza laboral, el Plan Maestro indica que el $4 \%$ de las personas que viven en Bogotá tienen su fuente de empleo fuera de la capital; en este mismo sentido, se proyecta para el año 2020 que el 8,25\% de los trabajadores vendrá de afuera, en tanto que el $7 \%$ trabajará afuera. De igual manera, el Plan Maestro encuentra que el eje Bogotá-Mosquera-Funza, cuyo transecto Bogotá-Mosquera conduce a Facatativá, es recorrido diariamente por 650.000 personas, inferior al eje Bogotá-Soacha en 50.000 personas y en 100.000 al Bogotá-Chía-Cajicá.

4.5 Tránsito Promedio Diario Semanal (TPDS). Esta faceta del fenómeno de metropolización se puede observar mediante el análisis de vínculos y flujos medidos a partir de los datos del Tránsito Promedio Diario Semanal capturados por el Instituto Nacional de Vías -Invías-. En la figura 1 se observan los transectos considerados para el análisis, mientras que la tabla 3 muestra la comparación de los TPDS de la Regional Núm. 12 de Cundinamarca entre los años 1989 y 1999. Para efectos de evidenciar cambios en la dinámica de metropolización, los TPDS se agruparon en rangos.

En la tabla anterior se observan transectos que ascendieron de categoría en el transcurso de diez años, indicando así un fuerte aumento en los vínculos que se dan entre Bogotá y los municipios contiguos, y entre ellos mismos (los transectos en negrilla han ascendido de categoría de TPDS). Por ejemplo, Bogotá-La Caro, que en 1989 se ubicaba en el rango 20.000-50.000, pasó en 1999 al rango mayores de 50.000, o Te del Salto-Fusagasugá, que pasó del rango 5.000-10.000 al de 10.000-15.000.

La tabla 4 muestra los porcentajes de variación que se presentaron en los mismos transectos para los dos años indicados anteriormente. Obsérvese que los mayores porcentajes de variación se presentan en las vías que comunican a Bogotá con los municipios contiguos, es decir, en el Primer Anillo Metropolitano.

32 Óscar Buitrago B. - Nohora I. Carvajal S. 
Figura 1. Mapa de vías de la regional Cundinamarca de Invías

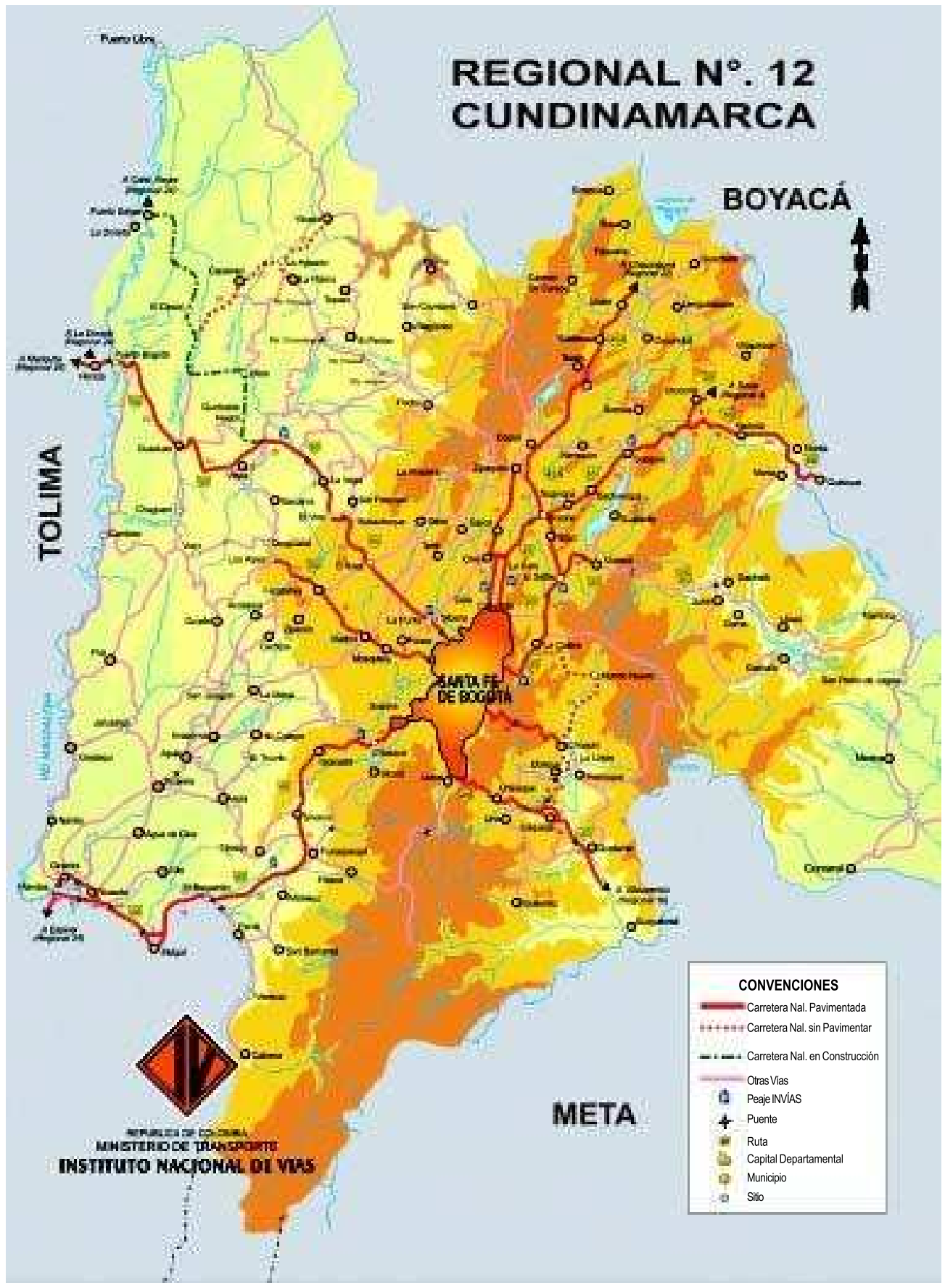

Perspectiva Geográfica Núm. 1 1, 2005 33 
Tabla 3. Comparación del Tránsito Promedio Diario Semanal 1989-1999

A partir de rangos para algunos transectos

del área de influencia de Bogotá

\begin{tabular}{|c|c|c|}
\hline $\begin{array}{l}\text { Tránsito promedio } \\
\text { diario semanal TPDS }\end{array}$ & SECTOR 1989 & SECTOR 1999* \\
\hline Mayor a 50.000 & Ninguno & Bogotá-La Caro \\
\hline Entre 20.000 y 50.000 & $\begin{array}{l}\text { Bogotá - La Caro, } \\
\text { Fontibón - Tres Esquinas }\end{array}$ & $\begin{array}{l}\text { Bogotá - Siberia, } \\
\text { Fontibón - } \\
\text { Tres Esquinas (Funza), } \\
\text { Mosquera - Tres Esquinas (Funza), } \\
\text { La Caro - Cajicá }\end{array}$ \\
\hline Entre 15.000 y 20.000 & Mosquera - Tres Esquinas & $\begin{array}{l}\text { La Caro - Briceño, Soacha - } \\
\text { Te del Salto, Madrid - Mosquera, } \\
\text { Puente El Cortijo - Siberia }\end{array}$ \\
\hline Entre 10.000 y 15.000 & $\begin{array}{l}\text { La Caro - Briceño } \\
\text { La Caro - Cajicá, Soacha - } \\
\text { Te del Salto, Madrid - } \\
\text { Mosquera, Facatativá - } \\
\text { Madrid, Puente El Cortijo - } \\
\text { Siberia }\end{array}$ & $\begin{array}{l}\text { Cajicá - Zipaquirá, Facatativá - Madrid, } \\
\text { Te del Salto - Fusagasugá, Siberia - } \\
\text { Cota, Briceño - Gachancipá, } \\
\text { Cota - Chía }\end{array}$ \\
\hline Entre 5.000 y 10.000 & $\begin{array}{l}\text { Sesquilé - Sisga, } \\
\text { Cajicá - Zipaquirá, } \\
\text { Fusagasugá - Melgar, } \\
\text { Melgar - El Paso } \\
\text { (vía a Girardot), } \\
\text { Tres Esquinas - Funza, } \\
\text { Te del Salto - Fusagasugá, } \\
\text { Siberia - La Punta, } \\
\text { Sisga - Chocontá, } \\
\text { Siberia - Cota, } \\
\text { Briceño - Gachancipá, } \\
\text { Cota - Chía }\end{array}$ & $\begin{array}{l}\text { Gachancipá - Sesquilé, } \\
\text { Sesquilé - El Sisga, } \\
\text { Te de Neusa - Ubaté, } \\
\text { Chusacá - Sibaté, } \\
\text { Fusagasugá - Melgar, } \\
\text { Melgar - El Paso (vía a Girardot), } \\
\text { Tres Esquinas (Funza) - } \\
\text { Funza (casco urbano), } \\
\text { Zipaquirá - Te de Neusa, } \\
\text { Siberia - La Punta, } \\
\text { La Punta - Te del Rosal, } \\
\text { Mosquera - Siberia, } \\
\text { Los Patios - La Calera, Cajicá - Tabio, } \\
\text { Sisga - Chocontá }\end{array}$ \\
\hline
\end{tabular}

Fuente: realizado por los autores a partir de datos suministrados por Invías (volúmenes de tránsito 1999). * Nombre asignado por Invías al transecto vial.

Al observar el comportamiento de este indicador, se debe destacar que la metropolización se produce y reproduce por la complejidad, flexibilidad y calidad de las redes, de tal manera que con el tiempo una mayor cantidad de centros urbanos será cobijada por el proceso de metropolización que genera un núcleo de mayor jerarquía.

34 Óscar Buitrago B. - Nohora I. Carvajal S. 
Tabla 4. Porcentaje de variación del TPDS entre 1989 y 1999

\begin{tabular}{|c|c|c|c|}
\hline SECTOR O TRANSECTO & 1989 & 1999 & VARIACIÓN \\
\hline CALLE 232 - LA CARO & 24.602 & 53.739 & 118,4 \\
\hline LA CARO - BRICEÑO & 11.257 & 15.246 & 35,4 \\
\hline BRICEÑO - SOPÓ & 3.066 & 3.907 & 27,4 \\
\hline GACHANCIPÁ - Sesquilé & 7.373 & 8.366 & 13,5 \\
\hline SESQUILÉ -SISGA & 6.362 & 6.937 & 9,0 \\
\hline LA CARO - CAJICÁ & 13.339 & 20.803 & 56,0 \\
\hline CAJICÁ - ZIPAQUIRÁ & 7.346 & 13.731 & 86,9 \\
\hline TE DE NEUSA - UBATÉ & 2.559 & 5.226 & 104,2 \\
\hline SOACHA - TE DEL SALTO & 14.239 & 19.091 & 34,1 \\
\hline CHUSACÁ - SIBATÉ & 1.849 & 5.182 & 180,3 \\
\hline FUSAGASUGÁ -MELGAR & 5.799 & 8.227 & 41,9 \\
\hline MELGAR - EL PASO & 5.950 & 8.673 & 45,8 \\
\hline FONTIBÓN - TRES ESQUINAS & 22.608 & 37.203 & 64,6 \\
\hline TRES ESQUINAS - FUNZA & 5.214 & 6.598 & 26,5 \\
\hline MOSQUERA - TRES ESQUINAS & 16.730 & 22.264 & 33,1 \\
\hline MADRID - MOSQUERA & 13.255 & 19.199 & 44,8 \\
\hline FACATATIVÁ - MADRID & 10.004 & 12.131 & 21,3 \\
\hline BRICEÑO - ZIPAQUIRÁ & 3.095 & 4.271 & 38,0 \\
\hline ZIPAQUIRÁ - TE DE NEUSA & 3.922 & 7.212 & 83,9 \\
\hline TE DEL SALTO - FUSAGASUGÁ & 7.254 & 11.122 & 53,3 \\
\hline MOSQUERA - LA MESA & 2.098 & 3.482 & 66,0 \\
\hline LOS PATIOS - LA CALERA & 4.357 & 6.315 & 44,9 \\
\hline CAJICÁ -TABIO & 3.559 & 7.056 & 98,3 \\
\hline PUENTE EL CORTIJO - SIBERIA & 11.975 & 21.645 & 80,8 \\
\hline SIBERIA - LA PUNTA & 5.725 & 11.276 & 97,0 \\
\hline LA PUNTA - TE DEL ROSAL & 4.313 & 7.909 & 83,4 \\
\hline SIBERIA - COTA & 5.153 & 13.174 & 155,7 \\
\hline MOSQUERA- SIBERIA & 4.724 & 6.853 & 45,1 \\
\hline BRICEÑO - Gachancipá & 8.663 & 11.232 & 29,7 \\
\hline COTA - CHÍA & 5.926 & 13.069 & 120,5 \\
\hline
\end{tabular}

Cálculos realizados por los autores.

4.6 Variable demográfica y volumen de carga transportada por vía terrestre. $\mathrm{El}$ estudio realizado por Óscar Alfonso (2002) presenta estas variables para identificar las modalidades y grados de metropolización.
- Variable demográfica: en la que se trabajan indicadores como 1) saldo con Bogotá, es decir, la diferencia entre emigrantes de Bogotá y emigrantes a Bogotá de los municipios de la sabana; 2) saldo sobre nacidos; 3) inmigrantes 
de Bogotá/residentes, y 4) índice de metropolización. De la revisión de estos indicadores se constata que Soacha muestra las relaciones más intensas con Bogotá; del total de los emigrantes de Bogotá a la totalidad de los municipios de la sabana, que es 139.377, los que se dirigen a Soacha, 69.294, representan la mitad $^{2}$. El saldo migratorio con Bogotá, en relación con el total de los nacidos en ese municipio, muestra una proporción tres veces mayor que el municipio que le sigue (Chía). El peso de los inmigrantes desde Bogotá sobre el total de los residentes del municipio es el más elevado, así como el índice de metropolización.

En un segundo estrato aparece un conjunto de siete municipios: Chía, Cota, Cajicá, Madrid, Funza, Tocancipá y Sibaté; es decir, los municipios más cercanos a Bogotá sobre los tres principales ejes viales: al norte, Chía, Cota y Cajicá; al occidente, Madrid y Funza, y al sur, Sibaté.

Un tercer estrato lo constituye el resto de los municipios de la sabana, entre los que se encuentran Facatativá y Zipaquirá, que muestran cierta autonomía al respecto.

Las modalidades de la metropolización en los diferentes grupos o estratos muestran que en Soacha la conurbación se ha profundizado, pues sus umbrales físicos no son en la actualidad muy evidentes y los mercados urbanos clave como el empleo, la vivienda y los servicios colectivos tienden a unificarse. Con respecto al segundo estrato, los municipios sobre el eje vial del norte también conforman la conurbación Chía-Cota-Cajicá, caracterizada por residencias semicampestres y la aparición de equipamientos metropolitanos como clubes y centros universitarios. Por temor a un proceso de ocupación del espacio similar al de Soacha, en los planes de ordenamiento territorial de estos municipios quedó estipulada la prohibición de fragmentar terrenos suburbanos en lotes de tamaño pequeño, potencialmente demandables por hogares de bajos recursos.

Los municipios conectados por el eje vial al occidente, de la misma manera, conforman una conurbación cuya particularidad principal tiene que ver con las implantaciones agroindustriales, en las que prevalece el cultivo de flores para la exportación, con gran atracción de mano de obra campesina, fundamentalmente femenina, dando lugar a una marcada densificación de los cascos urbanos. Por su parte, Sibaté es una continuación de la conurbación BogotáSoacha, cuya pauta de ocupación residencial es la de familias de bajos ingresos (Alfonso, 2002).

- Volumen de carga transportada por vía terrestre que tiene como destino y origen a Bogotá y la sabana: de acuerdo con el análisis realizado por Óscar Alfonso, con datos de 1993, el municipio de Mosquera presenta la

Datos del Censo Nacional de Población, 1993.

36 Óscar Buitrago B. - Nohora I. Carvajal S. 
mayor interacción con el mercado bogotano, debido a que cuenta con una estructura productiva diversificada, en la que se combina la agroindustria de flores con la producción de alimentos concentrados para animales, alimentos para humanos, construcción e industria de fundición. Soacha presenta el segundo valor del índice, pero su característica principal es la de una economía en retroceso. Facatativá, Subachoque, Sibaté, Funza y Sopó experimentan un índice similar al de Soacha; con la particularidad de que los dos últimos son importadores netos del mercado bogotano. Los municipios con menor importancia relativa para el mercado bogotano son Madrid, Tocancipá, Tenjo y Cota.

Concluye el autor que la interdependencia entre el proceso metropolitano de urbanización de la población y la dinámica metropolitana de la producción es bastante débil.

4.7 Relación entre el tamaño de predios rurales y la Unidad Agrícola Familiar $-\boldsymbol{U A F - 3}$ : de acuerdo con Absalón Machado, el tamaño promedio de la UAF en el departamento de Cundinamarca debe ser de cinco hectáreas; sin embargo, lo encontrado por Buitrago (2001) muestra que el tamaño real de los predios rurales en los municipios contiguos a Bogotá es mucho menor, en la mayoría de los casos menos de mil metros cuadrados $(0,1$ ha), "evidenciando un alto grado de suburbanización, que se expresa por la alta fragmentación de la tierra como resultado de la influencia directa del fenómeno de metropolización que actúa principalmente desde Bogotá, cuya tendencia es desplazar el uso agropecuario y en general el rural por actividades netamente urbanas".

4.8 Comportamiento de las rentas de la tierra: los precios de la tierra en los municipios contiguos a Bogotá presentan características y comportamientos diferenciados de acuerdo con factores relacionados con las actividades urbanas de vivienda, industria, comer-cio y servicios originadas desde el Distrito Capital hacia los municipios contiguos, configurando un territorio en donde se mezclan estas funciones urbanas con las tradicionalmente rurales; de este modo se observa una dispersión de las actividades urbanas a lo largo de las principales vías de comunicación de la sabana, causando el traslado de la renta absoluta urbana ${ }^{4}$ hacia espacios rurales y generando rentas diferenciales ${ }^{5}$ en estos (Buitrago, 2001).

UAF hace referencia a la cantidad mínima de tierra rural necesaria para producir dos salarios mínimos mensuales, que se supone sostienen una familia rural promedio.

4 Según Jaramillo S. (1994), la renta absoluta surge en los terrenos en los cuales ha tenido un fenómeno de convergencia espacial que ofrece ventajas excepcionales para realizar una gran cantidad de actividades, circunstancia que es difícil de reproducir a voluntad.

5 El mismo autor señala que las rentas diferenciales surgen de condiciones dispares que tienen los terrenos, por ejemplo, características físicas, intensidad del capital aplicado a la tierra, espacio construido y, en general, ventajas comparativas frente a otros predios. 


\section{Bogotá en la jerarquía ur- bana de Colombia}

La atracción que las ciudades ejercen sobre el territorio obedece a la capacidad que cada una de ellas tiene para facilitar el acceso a intercambios de bienes, servicios, ideas e información; las de mayor jerarquía son aquellas que polarizan funciones de alta especialización y, por tanto, en las que se expresa en mayor medida el fenómeno de metropolización. En el caso colombiano, los diferentes modelos explicativos de la jerarquía urbana coinciden en presentar a Bogotá en la primera categoría.

Así, el Ministerio de Desarrollo considerando variables relacionadas con las comunicaciones, la infraestructura y los servicios terciarios, estableció subsistemas de ciudades, a partir de lo cual se identificaron grandes regiones y subregiones (la jerarquía obtenida es de carácter funcional). El estudio basa su metodología en la teoría de los lugares centrales, según la cual cada una de las infraestructuras o servicios presentes en una ciudad constituyen una función urbana, y su mayor o menor cobertura espacial es lo que diferencia al centro urbano dentro de la red de ciudades.

La metodología consideró 18 tipos de servicios, cuya presencia o ausencia fue evaluada en cada una de las 1.006 cabeceras municipales observadas en el estudio. Estas variables permitieron la construcción de escalogramas, a partir de los cuales fue posible construir el índice urbano acumulado, que representa la combinación de atributos que posee un centro urbano, por lo cual muestra apropiadamente su importancia funcional relativa dentro del conjunto, así como su distancia funcional respecto de otros centros más o menos próximos.

A continuación se presenta la ubicación del área metropolitana de Bogotá dentro de esta jerarquía.

\begin{tabular}{|l|l|}
\hline \multicolumn{1}{|c|}{ JERARQUÍA } & \multicolumn{1}{c|}{$\begin{array}{c}\text { MUNICIPIOS DEL ÁREA } \\
\text { METROPOLITANA }\end{array}$} \\
\hline Centro metropolitano nacional & Bogotá \\
\hline Centros metropolitanos regionales & \\
\hline Centro regional mayor & \\
\hline Centro regional intermedio & Soacha \\
\hline Centro subregional mayor & $\begin{array}{l}\text { Facatativá, Fusagasugá, Zipaquirá, Funza, } \\
\text { Cota, Mosquera, Madrid, Chía, Cajicá }\end{array}$ \\
\hline Municipios restantes & \\
\hline
\end{tabular}

$\overline{6}$ Aportes para una nueva regionalización del territorio colombiano (ciudad y región en Colombia, 2001).

38 Óscar Buitrago B. - Nohora I. Carvajal S. 
Por su parte, el Instituto Geográfico Agustín Codazzi -IGAC- determinó la jerarquía urbana (1996), teniendo en cuenta el tamaño de los centros urbanos y la importancia de los equipamientos más especializados requeridos para un adecuado funcionamiento de las actividades terciarias, asociadas a la actividad urbana en la provisión de bienes y servicios. Esta investigación realizó la jerarquía urbana a partir del escalograma de Guttman (descrito por Rondinelli, 1988), que parte de considerar la presencia y cantidad de cada servicio determinado. El resultado de esta jerarquización identifica nueve niveles de centros urbanos que conforman la malla urbana nacional y un grupo de núcleos poblacionales asociados al campo, que es denominado "centros de servicios rurales" y corresponde a la mayoría de cabeceras municipales del país.

En esta jerarquización, el área metropolitana de Bogotá queda ubicada de la siguiente manera:

\begin{tabular}{|l|l|}
\hline \multicolumn{1}{|c|}{ JERARQUÍA } & \multicolumn{1}{c|}{$\begin{array}{c}\text { MUNICIPIOS DEL ÁREA } \\
\text { METROPOLITANA }\end{array}$} \\
\hline Metrópoli nacional & Bogotá \\
\hline Metrópoli regional & Bogotá \\
\hline Centros subregionales de primer orden & Bogotá \\
\hline Centros subregionales de segundo orden & Bogotá \\
\hline Centros subregionales de tercer orden & Bogotá \\
\hline Centros de relevo principales & Facatativá, Fusagasugá, Zipaquirá \\
\hline Centros de relevo secundarios & Madrid, Cajicá \\
\hline Centros locales principales & \\
\hline Centros locales secundarios & \\
\hline Centros urbanos básicos & \\
\hline
\end{tabular}

Para la Fundación Social, la red urbana colombiana es vista a partir del tamaño funcional y la especialización económica. En este estudio, la clasificación de las ciudades se realizó teniendo en cuenta el tamaño, las funciones que desempeñan y la especialización económica de estas, con el objeto de captar rasgos estructurales básicos de la red urbana colombiana. El tamaño funcional se define como un conjunto de fenómenos asociados a la talla y a las funciones de las ciudades según la teoría del lugar central (Christaller). A partir de este concepto se realizó un análisis de especialización económica y concentración del empleo. La ciudad es considerada una unidad socioespacial básica de soporte de la producción cultural, de la innovación social y de la actividad económica del mundo 
contemporáneo. Tales características hacen que asuma la centralidad como forma generalizada y ejerzan una función de dominio. Las redes de ciudades dan lugar a una jerarquía y a una subordinación-complementariedad entre los asentamientos.

A partir de la anterior conceptualización, se obtuvo el Índice de Tamaño Funcional y se clasificaron los municipios en siete grupos (análisis de conglomerados). Luego, se utilizó el Coeficiente de Localización para captar diferencias en las estructuras económicas y precisar ramas más destacadas (cinco tipos de municipios) y el Índice de Concentración para determinar en qué ciudades existe una participación mayor a la esperada de una actividad.

A continuación se presenta el área metropolitana de Bogotá en esta categorización de tamaño funcional:

\begin{tabular}{|l|l|}
\hline JERARQUÍA & $\begin{array}{l}\text { MUNICIPIOS DEL ÁREA } \\
\text { METROPOLITANA }\end{array}$ \\
\hline Centro metropolitano nacional & Bogotá \\
\hline Centro metropolitano regional & \\
\hline Centro regionales mayores & Soacha \\
\hline $\begin{array}{l}\text { Centro regional intermedio y } \\
\text { municipios que forman parte } \\
\text { de áreas metropolitanas }\end{array}$ & $\begin{array}{l}\text { Chía, Facatativá, Fusagasugá y } \\
\text { Zipaquirá }\end{array}$ \\
\hline Centro subregional mayor & \\
\hline Municipios restantes & \\
\hline
\end{tabular}

6. Directrices y orientaciones para el ordenamiento territorial (DOT) del departamento de Cundinamarca en relación con la expansión de Bogotá

Al ser Bogotá no solamente la capital del país, sino también del departamento de Cundinamarca, y al tener su área de influencia sobre territorio cundinamarqués, se hace necesario mostrar las directrices y orientaciones para el ordenamiento territorial que el departamento estableció con referencia al fenómeno metropolitano.

Estas directrices y orientaciones constituyen el marco de referencia general para la formulación de los POT municipales del departamento. Entre otros principios, se hace referencia a que

... las DOT determinan acciones territoriales que requieran la acción conjunta con el Gobierno Nacional,

40 Óscar Buitrago B. - Nohora I. Carvajal S. 
el Departamento, el Distrito Capital de Bogotá y otros entes territoriales[...] Están concebidas para alcanzar un consenso entre las diversas administraciones y entidades públicas y privadas que actúan en el departamento, así como entre los habitantes de Cundinamarca con respecto a la definición del modelo territorial cundinamarqués [...] Son de carácter vinculante y por tanto de obligatoria aplicación en los POT (Gobernación de Cundinamarca, 1999).

En el capítulo IV, sobre Expansión de Bogotá hacia el territorio de Cundinamarca, las DOT reconocen que ante el crecimiento de la capital se debe dar respuesta desde el departamento, considerando como prioritario atender, planear y resolver de manera compartida los problemas derivados de esta tendencia, a través de actividades de planeación coordinadas entre las diferentes entidades administrativas y de la concertación entre el Distrito Capital y los municipios circundantes, para establecer usos del suelo compatibilizando el espacio ciudad-región (Gobernación de Cundinamarca, 1999).

\section{Visión regional de los planes de ordenamiento territorial de los municipios del área de influencia de la sabana}

El estudio de la construcción histórica del territorio, del origen de la primacía urbana de Bogotá, junto con el análisis de algunos indicadores de metropolización y de las jerarquías urbanas, sustenta ampliamente la complejidad del fenómeno metropolitano. Además, la revisión y análisis de la práctica de la planificación de los municipios del área metropolitana y su expresión en los planes de ordenamiento territorial conduce a demostrar que este fenómeno aún no es dimensionado en su verdadera magnitud por los actores de la planificación municipal.

Para comprender dicha práctica se revisaron y analizaron los documentos de los POT, considerando la visión, objetivos, formulación, estrategias e instrumentos consignados en algunos de ellos, buscando identificar ideas concretas acerca de la concepción y proyección de las relaciones entre cada municipio y Bogotá, como la ciudad núcleo o principal del área metropolitana.

A continuación se presentan los resultados, partiendo del POT de Bogotá y siguiendo con una muestra de municipios tomada de cada anillo metropolitano.

\section{Visión metropolitana en el POT de Bogotá D. C.}

\section{Contexto del plan}

De acuerdo con el Documento Diagnóstico del POT, en Bogotá y la sabana de Bogotá se presenta una alta 
dinámica de crecimiento urbano, descrito principalmente por la cantidad de hectáreas ocupadas por la ciudad y los cascos urbanos de los municipios sabaneros y por un incremento notorio de usos urbanos, entre los que se hace especial énfasis en la conurbación con los municipios vecinos de Cota, Chía, Cajicá y La Calera.

En el tema urbano regional, el documento diagnóstico trata dos tópicos principales: la sabana, como un territorio de la cuenca alta del río Bogotá, y el crecimiento urbano regional.

Con respecto al primero, se describe la sabana de Bogotá como un territorio integrado a la cuenca del río Bogotá "en términos físicos, por las tierras planas, los cerros que la limitan al oriente y occidente hasta la divisoria de aguas, los páramos y las cuencas de los afluentes del río...". También caracteriza a la red de asentamientos urbanos de la sabana con un gran centro metropolitano y una estructura radial de asentamientos menores vinculada a la red vial nacional y regional. Bajo este mismo tópico se hace una breve descripción de la evolución del uso agrícola, señalando un significativo aumento de la praderización, disminución de las tierras destinadas a la producción agrícola, incremento poco significativo de las tierras destinadas a la horticultura y aumento discreto de las tierras en floricultura.

En cuanto al aspecto urbano regional, se identifica una dinámica que no ha favorecido la integración y articulación del espacio regional, y que según el POT ha derivado en problemas tanto para Bogotá como para los municipios del área de influencia, manifiestos en desequilibrios espaciales en la oferta de empleo y en la distribución de equipamiento. De acuerdo con el documento del POT, el proceso de urbanización de la ciudad ha mantenido un modelo espacial concentrado en la ciudad principal, con una alta densidad de población. El modelo de evolución de Bogotá se explica principalmente por el proceso de conformación del sistema de ciudades en el país (descrito en el contexto histórico de este documento), el cual muestra que hasta hace algunos años se presentaba una cuadricefalia originada principalmente por la forma desarticulada en que se construyeron las regiones en el país; sin embargo, esta tendencia ha cambiado en las últimas décadas, debido a la fuerte concentración de actividades altamente especializadas en Bogotá, haciendo que su jerarquía urbana se aleje de las otras tres ciudades (Medellín, Cali y Barranquilla).

Para el POT, la región urbana de Bogotá se encuentra en proceso de consolidación, ya que asume y afianza funciones de escala nacional y regional, así como manifiesta una participación en actividades industriales, agroindustriales y comerciales, en la oferta de servicios educativos y en el desarrollo de actividades recreativas y turísticas.

42 Óscar Buitrago B. - Nohora I. Carvajal S. 
La consolidación de la región urbana se manifiesta principalmente por la presencia de un núcleo urbano central, dos centros regionales en los bordes norte y occidente (Zipaquirá y Facatativá), tres conurbaciones (CotaChía-Cajicá, Soacha-Bogotá y FunzaMosquera-Madrid) y la ocupación por usos urbanos de los espacios periurbanos.

\section{Determinantes del modelo de ordenamiento territorial}

En las políticas del Plan relacionadas con la sostenibilidad del desarrollo ambiental y económico, se expresa la necesidad de construir un territorio regional, considerando que este tema es básico en la concertación del Distrito con el departamento de Cundinamarca y los municipios vecinos. En este sentido, el POT establece la conveniencia de desarrollar propuestas en torno a un modelo territorial regional y un proyecto de agenda marco para avanzar en este campo con las entidades involucradas. Se propone como instrumento de planificación y gestión un proyecto regional concertado que facilite la coordinación entre la Corporación Autónoma Regional de Cundinamarca, el departamento de Cundinamarca, el Distrito Capital y los municipios de la región, todo dentro del propósito de promover el manejo integrado de la sabana, en la búsqueda de nuevas oportunidades para el desarrollo regional.
El modelo regional parte de considerar que el territorio sabanero está constituido por tres espacios regionales articulados por un sistema de relaciones que facilitan y potencian la interacción socioespacial. Ellos son: el espacio regional protegido, el espacio regional agrícola y el espacio regional urbano. Las determinaciones por considerar en el ordenamiento general de la región son:

- Delimitación del área o suelo regional de protección: se propone un modelo regional de la Estructura Ecológica Principal, contemplándose una articulación del Distrito con el ámbito regional a partir de un sistema de áreas protegidas, que cobija varios elementos ya definidos como zonas de protección, en proceso legal de declaración o en proceso de delimitación. En la estructura ecológica regional se proponen áreas para la recuperación de cuerpos de agua, la preservación del sistema montañoso, la implementación de parques naturales y plantas de tratamiento para los ríos Bogotá, Fucha, Juan Amarillo y Tunjuelito.

- Definición del espacio agrícola regional: se concibe como un espacio para el control del crecimiento de los espacios urbanos y de la especulación del suelo rural; está vinculado a la preservación y mejoramiento de las condiciones ambientales de la sabana.

- Ordenamiento del espacio destinado a funciones urbanas $y$ metropolitanas: 
se propone reconocer el sistema actual de asentamientos y ordenarlo con la mira puesta en la concentración de la urbanización, para evitar el nacimiento de nuevos asentamientos. Se propone un uso intensivo del suelo en la ciudad, para evitar que esta se vuelque a las zonas rurales, especialmente en aquellos espacios de urbanización dispersa. De igual manera, se afirma que es necesario evitar crecimientos espontáneos urbanos. El sistema de asentamientos de la región debe complementarse con un sistema vial de comunicaciones en forma de anillos. Los elementos naturales y ambientales deben fortalecerse para controlar el crecimiento de la ciudad. También se recomienda diversificar las ofertas de localización para los grupos familiares y para las actividades económicas.

- Definición de los sistemas relacionales que permiten la integración e interacción de los espacios regionales: se recomienda solidificar los sistemas de infraestructura y redes para fortalecer las funciones y el ordenamiento de cada espacio regional. Este componente debe favorecer las interacciones, relaciones e intercambios regionales y suprarregionales. Los sistemas concebidos son el vial, el de saneamiento básico, el de servicios domiciliarios y el de equipamiento (educación, salud, recreación y productivo), con prioridad en los centros regionales y las conurbaciones.

\section{Visión metropolitana en el POT de algunos municipios del Primer Anillo}

\section{Soacha}

\section{Contexto del municipio}

Esta municipio se encuentra localizado en el extremo sur la sabana de Bogotá y se caracteriza, principalmente, por el fuerte desarrollo y concentración de vivienda subnormal, una zona industrial ya consolidada en el sector de Alicachín y una zona de haciendas con enorme potencial agrícola (CIDER, 2000).

El documento del POT fue estructurado en tres componentes: uno de Valoración y Formulación, otro de Dimensiones y el tercero de Atributos. Siguiendo ese orden, a continuación se presenta el análisis de la visión metropolitana plasmada en él.

\section{Valoración y formulación}

En el documento técnico soporte del POT de este municipio, en la metodología para determinar la demanda ambiental, se encuentra una visión de planificación cerrada expresada de la siguiente manera: "La demanda analizada para el municipio de Soacha fue la de servicios, teniendo en cuenta las necesidades de la población, sus tendencias y perspectivas al interior del municipio".

44 Óscar Buitrago B. - Nohora I. Carvajal S. 
De otra parte, la fundamentación del modelo de desarrollo económico para el municipio de Soacha parte de considerar, por un lado, su proyección como una ciudad del siglo XXI enmarcada en el fenómeno de la globalización, y, por otro, que una de sus características es la degradación en múltiples sentidos de la población, debida no solamente a la acción local, sino también a la presión conjunta de todo el país, que la ha constituido en amortiguador para el fenómeno nacional del desplazamiento y en general de las migraciones de todo tipo.

Así mismo, este modelo de desarrollo económico tiene en cuenta la presencia de una asimetría laboral en la relación Soacha-Bogotá, manifiesta en que su abundante mano de obra no calificada es absorbida por la capital, convirtiendo a Soacha en ciudad dormitorio y propiciando el menor consumo de bienes y servicios en este municipio.

Por otra parte, en el análisis de competitividad se considera que una de las ventajas del municipio es su localización estratégica, al constituirse como bisagra entre Bogotá y el occidente del país; de igual manera, como puerta de acceso a la capital, comparte el transporte de la Autopista Longitudinal de Occidente (ALO), la Avenida Ciudad de Cali, la Autopista al Llano, la Mondoñedo, la Circunvalar de Oriente y la Avenida Terreros, avenidas que estructuran la red vial de la ciudad. El mismo análisis considera que la cercanía del municipio a Bogotá, expresada en una conurbación, constituye un factor que favorece el desarrollo de su economía, al poder aprovechar las ventajas en materia de vías (sistema de transporte integrado Transmilenio), aeropuerto internacional, telecomunicaciones, centros financieros, servicios públicos, instituciones y desarrollo cultural, entre otros.

Otro elemento del análisis de competitividad en materia regional es el concerniente a la oferta que el municipio hace de materiales de construcción (arena, piedra, ladrillo) y a la demanda de estos por la ciudad capital, relación considerada impulsora de la producción y el empleo municipales.

\section{Dimensiones}

El diagnóstico del POT concluyó que debido al acelerado proceso de metropolización del Distrito Capital y, más exactamente, a las restricciones de área que tiene para expandirse dentro de su perímetro urbano, su población se ha venido ubicando en aquellos municipios aledaños en donde, como es el caso de Soacha, el acceso a la vivienda por parte de las familias de ingresos medios es más fácil.

En el tema de la salud de la población rural, el diagnóstico concluye que el $92,3 \%$ se encuentra desprotegida $\mathrm{y}$ tiene que recurrir para asistencia básica y urgencias al casco urbano del municipio o viajar a Bogotá. 
Continuando con lo plasmado en el diagnóstico, se manifiesta que la metropolización ha favorecido la localización de la industria en Soacha, propiciada además por las políticas de Estado que han buscado desconcentrar esta actividad de las grandes ciudades y favorecer su implantación en ciudades intermedias como Soacha, y por la oferta de terrenos a bajos precios.

\section{Atributos}

El enfoque conceptual de la Visión Urbano Municipal considera al municipio en un contexto regional, en el que se presentan relaciones funcionales de jerarquía, dependencia y estructuras espaciales como el sistema vial, la red urbana, los nexos familiares, el intercambio y demanda de insumos y productos, entre otros.

\section{Chía}

\section{Contexto del municipio}

Este municipio, contiguo al norte de Bogotá, fue perdiendo su vocación agrícola en las últimas décadas debido, por un lado, al traslado desde la capital de una serie de funciones urbanas como servicios educativos y vivienda para estratos socioeconómicos altos y, por otro, a la especialización de un nuevo tipo de producción agroindustrial, con uso intensivo del suelo y destinada al mercado externo (floricultura).

Del documento de Acuerdo Núm. 17 del 2000, "Por el cual se adopta el Plan de Ordenamiento Territorial del Municipio de Chía", se extraen las siguientes características, que muestran la visión metropolitana allí plasmada. Se observa en el planteamiento de las políticas, objetivos y estrategias, la conciencia de que Chía forma parte de la región metropolitana de Bogotá.

\section{Política y objetivos regionales}

Se considera fundamental la "Inserción del municipio de Chía, como centro prestador de servicios, en el modelo agropolitano $^{7}$ propuesto para la región, soportado en su posición estratégica, sus fortalezas sociales, culturales y productivas y en su autonomía”.

Una característica del documento de Acuerdo de Chía es que plantea un objetivo regional, que se formula de la siguiente manera: "Hacer de Chía el municipio líder en el área de influencia por el aprovechamiento de sus ventajas competitivas: geoespaciales, sociales, culturales y productivas y por la calidad de vida de sus pobladores".

7 La agrópolis se plantea como alternativa de "las ciudades grandes". Sintetiza el acople estructural entre lo urbano y lo rural. Por definición, ofrece el paradigma de una ciudad regional: un agro recuperado para su función productiva y una configuración urbanística multicéntrica. Es polinucleada y forma un conjunto de ciudades y pueblos jerarquizados por una metrópolis (Mendoza, 1999). El objetivo de la agrópolis está en organizar integralmente una región geográfica, como simbiosis estructural de agro y ciudad, donde los actores son campesinos y ciudadanos organizados (Convenio CARSGC, 1996).

46 Óscar Buitrago B. - Nohora I. Carvajal S. 
Como objetivo específico relacionado con la temática regional, se plantea que la explotación y protección de la tierra y los recursos naturales del municipio deben complementarse con los usos de la gran ciudad y de los municipios circundantes, con criterios de desarrollo compartido con el entorno regional.

\section{Estrategias para el ordenamiento territorial}

Se incorpora el concepto de equilibrio biorregional, referido al uso sostenible y sustentable del medio natural del municipio en relación con la región.

Con respecto a las estrategias para la integración regional, el municipio propone concertar con el Distrito Capital y con los municipios circunvecinos la construcción del modelo de desarrollo regional, dentro de criterios de complementariedad y subsidiaridad -devenidos de los principios constitucionales-, e impulsar la construcción de una red regional interinstitucional (UMATAS, SENA, CAR, otras) para la prestación de los servicios públicos y la recuperación, conservación y protección ambiental.

\section{Visión metropolitana en el POT de algunos municipios del Segundo Anillo}

\section{Cajicá}

\section{Contexto del municipio}

El municipio de Cajicá pertenece a la cuenca del río Bogotá, se encuentra al norte de la sabana de Bogotá, cuenta con una vocación agropecuaria; sin embargo, en los últimos años ha presentado una diversificación en la actividad económica, destacándose un incremento acelerado en el sector industrial y comercial.

\section{Objetivos del POT}

En la formulación de objetivos para el ordenamiento territorial de este municipio, se establece la necesidad de construir equipamientos e infraestructuras de cobertura regional para propiciar el desarrollo de la actividad manufacturera, agrícola, de servicios y el desarrollo económico general del municipio.

\section{Diagnóstico}

En el Informe de Diagnóstico, Cajicá se considera municipio dormitorio, es decir, que gran parte de sus pobladores desarrollan sus actividades diarias en Bogotá y regresan en la noche.

En el Acuerdo 08 del 30 de julio del 2000, por el cual se adopta el Plan Básico de Ordenamiento de Cajicá, una de las estrategias formuladas para garantizar la consecución de los objetivos es la del fortalecimiento de la gobernabilidad a través, entre otros, de potenciar el liderazgo provincial y regional de Cajicá dentro de la Asociación de Municipios de Sabana Centro (Asocentro), concretando:

- propuesta de proyectos regionales, 
- fortaleciendo la interlocución de la región con el departamento y la nación $\mathrm{y}$

- promoviendo la planificación conjunta con los demás municipios de Asocentro.

Como política para el fortalecimiento de la gobernabilidad, se plantea que Cajicá debe tomar la iniciativa de presentar ante el Congreso de la República, y como producto de la concertación, un proyecto de ley para la constitución de la Provincia de Sabana Centro.

\section{Bojacá}

\section{Contexto del municipio}

Municipio localizado en el occidente de la sabana de Bogotá; se caracteriza por un predominio de la actividad agrícola tradicional y la agroindustria de las flores, así como por el turismo religioso de fin de semana, por las continuas peregrinaciones a la Virgen de Bojacá.

La localización en este municipio del botadero de Mondoñedo, como infraestructura de servicios a escala metropolitana y regional, presenta una problemática ambiental por resolver.

\section{Objetivos y formulación del POT}

En el objetivo general no se hace alusión al tema, mientras que en uno de los objetivos específicos se establece como necesario comprobar el funcionamiento del sistema vial del municipio y su integración a la red regional.

En la formulación del POT se considera al municipio como una región geográfica, de tal manera que los componentes físicos, bióticos y socioeconómicos interactúan y conforman un macrosistema regional, indicando que se tiene una visión cerrada del municipio. Contrario a lo anterior, en el mismo documento se expresa que existe un estrecho vínculo de este municipio con Facatativá, más que con Bogotá.

\section{Visión metropolitana en el POT de algunos municipios del Tercer Anillo}

\section{Subachoque}

\section{Contexto del municipio}

Municipio localizado al noroccidente de la sabana de Bogotá; con alto potencial agrícola y ganadero, es una de las despensas de la capital. Por sus características de alta calidad ambiental y paisajística, familias de estratos socioeconómicos altos de Bogotá tienen allí sus fincas veraniegas; además, constituye uno de los más importantes destinos del turismo de fin de semana de los bogotanos.

\section{Documento de Acuerdo del POT}

En el documento del Acuerdo por el cual se adopta el POT municipal, en el

48 Óscar Buitrago B. - Nohora I. Carvajal S. 
Componente Rural se destacan como ventajas comparativas algunas relaciones que establece Subachoque con Bogotá: primero, la situación geográfica del municipio, próxima al Distrito Capital y a los centros de mercadeo y producción de bienes y servicios, lo que constituye una de sus mayores fortalezas; segundo, la infraestructura vial del municipio y su interconexión con vías de importancia regional garantizan la salida de sus productos a los mercados y la posibilidad de ingreso de un volumen importante de turistas, $\mathrm{y}$, por último, la calidad de sus suelos y su uso en actividades agropecuarias garantizan una producción importante en la provisión de alimentos para la capital y la sabana de Bogotá.

\section{El Rosal}

\section{Contexto municipal}

Localizado al occidente de la sabana de Bogotá y atravesado por la principal vía que comunica a Bogotá con Medellín y el norte del país, es uno de los últimos municipios constituidos, al haberse segregado de Subachoque. Su principal vocación es la agricultura.

\section{Formulación y estrategias del POT}

En este documento se hace una somera alusión al tema regional, en la que intenta identificar vínculos con los municipios de la Sabana Occidente en la dimensión ambiental, económica y social. La cercanía a Facatativá le ofrece ventajas en el mercadeo de productos agrícolas y pecuarios. Como consecuencia de la cercanía con la Autopista Medellín, existe una amplia infraestructura de restaurantes y sitios turísticos que se han convertido en lugar de recreo los fines de semana para muchos habitantes de la capital y del país.

Como estrategia se plantea la necesidad de observar coherencia con su entorno regional, para lo cual deberá: primero, establecer mecanismos de concertación con la capital y los municipios vecinos; segundo, atender los principios de concurrencia, subsidiariedad y coordinación administrativa, y tercero, establecer mecanismos para definir zonas de protección y conservación, manejo de residuos sólidos y matadero regionales.

\section{Facatativá y Zipaquirá}

Estos municipios se localizan en el borde noroccidental de la sabana de Bogotá, dinamizando y articulando las relaciones de la región metropolitana con los municipios de la vertiente occidental de la cordillera Oriental.

Tanto para Facatativá como para Zipaquirá, considerados Centros de Relevo Principal ${ }^{8}$ de la sabana de Bogotá, se reconoce una fuerte influencia de la metropolización de la

8 Ver en este mismo documento Jerarquía Urbana de Colombia realizada por el IGAC. 
ciudad capital, que estos municipios se proponen contrarrestar con proyectos de especialización de servicios, buscando consolidar su propia área de influencia dentro de esta gran área metropolitana.

\section{Contexto de Facatativá}

Facatativá se localiza en el borde occidental de la sabana de Bogotá, situación privilegiada debido a que cuenta con abundante recurso hídrico que abastece los municipios de Sasaima, Albán, La Vega y San Francisco, en la vertiente occidental de la cordillera Oriental, y los municipios de Sabana de Occidente.

Políticas, objetivos y estrategias generales de carácter regional del POT de Facatativá

En las políticas del POT se busca consolidar el municipio como centro jerárquico regional, aprovechando $\mathrm{y}$ potencializando sus valores culturales, patrimonio ambiental, oferta comercial, turística y educativa que afiancen al municipio como centro de servicios y de industria con carácter regional y nacional.

En los objetivos regionales del POT se busca establecer políticas y directrices que propicien la consolidación de una Unidad Regional, conjuntamente con los municipios del área de influencia de Facatativa, que mejore el aumento de la productividad y calidad de vida de los habitantes.
En el Modelo Regional del POT municipal se propone buscar la construcción y consolidación de un modelo regional sostenible, para lo cual se determinan las siguientes políticas:

- Promover una concertación regional con los municipios vecinos en asuntos como: manejo ambiental; consolidación de un sistema jerarquizado de asentamientos; identificación, construcción y mantenimiento de los sistemas regionales de abastecimiento de aguas, saneamiento y energía eléctrica; identificación, construcción y mantenimiento de la red vial y de transporte; identificación de proyectos estratégicos de interés común para la región, e incorporación de un enfoque integral sobre el sistema hídrico del río Bogotá, orientado a su adecuado manejo y protección, reconociendo su impor-tancia como elemento básico de la estructura ecológica principal de la región.

- Dar respuesta a los requerimientos de la región en términos de la localización de estructuras que permitan consolidar los actuales procesos de producción, el favorecimiento para la localización de nuevos servicios a la vivienda y la industria y el incremento de las áreas de recreación.

- Complementar la intensa oferta educativa, a través de la localización de equipamientos culturales, el mejoramiento del sistema de movilidad y el incremento de las áreas recreativas y deportivas.

50 Óscar Buitrago B. - Nohora I. Carvajal S. 


\section{Contexto de Zipaquirá}

Zipaquirá forma parte de un corredor geográfico con dirección noroccidental (Chía, Cajicá, Zipaquirá, Cogua, Nemocón, Tausa y Sutatausa) que permite intercambios entre la sabana de Bogotá y los valles de UbatéChiquinquirá y río Negro, este último localizado en la vertiente occidental de la cordillera Oriental. Históricamente se caracteriza por una intensiva producción agrícola, pecuaria y de recursos minerales, como la sal y el carbón.

La relación de Zipaquirá con el Distrito Capital se caracteriza por la fuerte polaridad que ejerce este sobre el territorio sabanero, a pesar de haber logrado mantener una relativa autonomía derivada de su carácter como centro de servicios e intercambios dentro de la región sabanera. Como contrapeso a esta fuerte polaridad, asociaciones municipales como Asocentro (Asociación de Municipios Sabana Centro), de la cual Zipaquirá se constituye como centro organizador de Cajicá, Cota, Gachancipá, Sopó, Tenjo, Cogua, Chía, Nemocón, Tabio y Tocancipá, han unificado acciones en torno a los intereses de los municipios que rodean a Bogotá9

Políticas, objetivos y estrategias regionales del POT de Zipaquirá
De acuerdo con la política definida por el POT, Zipaquirá debe consolidar el liderazgo regional como parte de Asocentro, para de este modo mantener su autonomía y evitar ser absorbida definitivamente por la metrópoli.

Como estrategia para tal fin, el POT fijó la promoción de proyectos regionales diseñados en función del beneficio directo de los municipios de Asocentro, independientemente de las directrices de manejo de la región establecidas por la metrópoli.

A pesar de lo anterior, en el documento de Acuerdo del POT, en el artículo relacionado con políticas, objetivos y estrategias de fortalecimiento de Zipaquirá como polo regional, las políticas y estrategias regionales no se ven plasmadas.

\section{Visión metropolitana en el POT de algunos municipios de la Sabana Periférica}

\section{Fusagasugá}

\section{Contexto del municipio}

Municipio localizado al suroccidente de Bogotá, en la vertiente occidental de la cordillera Oriental; no hace parte de la sabana de Bogotá, sin embargo,

Documento Técnico de Soporte del POT de Zipaquirá. Formulación. Julio de 1999. 
desde la perspectiva de este trabajo, por sus intensas relaciones con la capital, es considerado parte de la periferia de la región metropolitana de Bogotá.

En Fusagasugá se refleja la situación nacional en lo que tiene que ver con el orden público y la economía. El comercio, principal actividad del municipio, se ha visto afectado por la presencia de grupos al margen de la ley. Al mismo tiempo, el principal problema del municipio es el desempleo ${ }^{10}$.

Regionalmente, Fusagasugá es el centro económico de nueve municipios (Provincia de Sumapaz): Granada, Tibacuy, Pasca, Arbeláez, Pandi, Venecia, San Bernardo, Cabrera y Silvania, y la frontera cultural entre los departamentos de Tolima y Cundinamarca. Así mismo, es una ciudad prestadora de servicios, especialmente el de educación (en Fusagasugá el mayor porcentaje de población es menor de 25 años).

Visión, objetivos, estrategias y políticas de largo y mediano plazo para el manejo del territorio

El documento Técnico de Formulación del POT presenta como visión municipal "una ciudad prestadora de servicios" para la región, el departamento y el país, por sus características sociales, culturales, económicas, ubicación estratégica, desarrollo urbanístico, equipamiento en salud y educación y por su condición de cabecera de provincia.

A pesar de que es un municipio altamente vinculado a Bogotá, como se demuestra con los indicadores de metropolización explicados arriba, la visión, objetivos, estrategias y políticas para el manejo del territorio consideran a Fusagasugá como centro de la provincia de Sumapaz, sin tener en cuenta la relación con la región metropolitana de la capital, idea que se afianza con lo expresado por el director de Planeación Municipal $(2002)^{11}$, para quien el modelo de ordenamiento territorial municipal propuesto no fue el más acertado, por cuanto no se consideró la visión regional con la sabana de Bogotá.

\section{Conclusiones}

El carácter coordinado de la planificación de una región metropolitana puede concebirse a partir de relaciones horizontales, verticales y temporales ${ }^{\mathbf{1 2}}$. Por horizontalidad se hace referencia a la concurrencia y armonía de las distintas políticas sectoriales que se desarrollan en el territorio; por verticalidad, a la acción concurrente

\footnotetext{
10 Basado en la entrevista realizada al jefe de planeación municipal en octubre de 2002.

11 Trabajo de campo realizado en este municipio en octubre de 2002.

12 Aspectos que son formulados por Massiris (1997) para mostrar el carácter prospectivo y coordinado de los planes de ordenamiento territorial.
}

52 Óscar Buitrago B. - Nohora I. Carvajal S. 
y armónica entre los distintos niveles territoriales que tienen jurisdicción administrativa sobre el territorio, que implica no solamente la conveniencia de las decisiones de carácter regional, sino la incidencia de las nacionales sobre las locales y regionales, y por coordinación temporal se hace referencia a la continuidad en el tiempo de la política ordenadora, aunque cambien los gobiernos.

En cuanto a las relaciones horizontales, en este estudio se revisó la concurrencia y armonía de la política regional para el área metropolitana de Bogotá en cada POT, encontrando que:

- El POT de Bogotá considera la ciudad como núcleo del área metropolitana que se circunscribe únicamente a la sabana de Bogotá, sin incluir otros municipios que están recibiendo igual influencia que los sabaneros, pero localizados en las vertientes de la cordillera Oriental, como el caso de Fusagasugá. Bogotá reconoce desequilibrios espaciales en el interior de la región en la oferta de empleo y la localización de equipamientos.

- Los POT de Fusagasugá, Facatativá, Zipaquirá y Cajicá visionan su respectivo municipio como centro de una región conformada alrededor de ellos, sin tener en cuenta en los procesos de planificación del territorio la articulación con Bogotá como metrópoli. Como estrategia para buscar contrarrestar la polarización de
Bogotá, municipios como Facatativá y Zipaquirá proponen consolidar su propia área de influencia. En esta búsqueda se presentan disputas por adquirir el liderazgo (centralidad) en sus respectivas regiones, como en el caso de Zipaquirá y Cajicá en relación con la Asociación de Municipios del Centro de la Sabana (Asocentro).

- Municipios como Chía y Subachoque se reconocen como parte del área de influencia metropolitana de Bogotá. En el caso de Chía se propone el complemento de usos del suelo con la gran ciudad y los municipios vecinos.

- Municipios como Bojacá manifiestan tener mayores vínculos con centros subregionales de segundo orden (Facatativá) que con la capital.

- El Rosal, a pesar de reconocer su pertenencia a la Sabana Occidente, polarizada por Facatativá, propone concertar con la capital algunos temas de carácter regional.

En cuanto a las relaciones verticales, se identificó una primacía de los intereses municipales sobre la necesidad de planificar una región evidentemente metropolitana; a excepción del POT de Bogotá, en el que se propone la concertación con los municipios y el departamento de Cundinamarca para construir un modelo territorial regional y un proyecto de agenda marco. Sin embargo, se considera únicamente a los actores institucionales, en este caso el 
Distrito Capital, la Corporación Autónoma Regional de Cundinamarca y las administraciones municipales, sin tener en cuenta los demás actores que deben ser involucrados en la planificación del territorio. Por otra parte, las directrices y orientaciones dadas por el departamento de Cundinamarca no fueron consideradas en la mayoría de los casos, ignorando los niveles de prevalencia en las determinaciones del ordenamiento territorial.
Referente a la temporalidad, las pocas políticas de los POT municipales relacionadas con el tema de la región metropolitana de Bogotá, formuladas para el mediano y largo plazo ${ }^{13}$, no darán solución real a la problemática del área de influencia de la capital, pues, como se menciona en el análisis de horizontalidad, se presenta una diversidad de visiones de la planificación a escala metropolitana, sin llegar a puntos de encuentro que conduzcan a procesos integrales de ordenación del territorio $\mathrm{y}$, en últimas, al mejoramiento de la calidad de vida de la creciente población del área metropolitana.

\section{Bibliografía}

AlBEt, A y P. Benejam (2000): Una geografia humana renovada: lugares y regiones en un mundo global. España: Vicens Vives.

Alcaldía Cívica de Facatativa (2002): Plan de Ordenamiento Territorial. Facatativá 20012021. Oficina Asesora de Planeación Municipal. Facatativá.

Alcaldía Mayor de Bogota D. C. (1994): Misión siglo XXI. El futuro de la capital.

, Departamento Administrativo de Planeación Distrital (2000): Decreto 619 de 2000. Plan de Ordenamiento Territorial. Bogotá.

Alcaldía Municipal de Cajicá (2000): Acuerdo 08 del 30 de julio del 2000, por el cual se adopta el Plan Básico de Ordenamiento. Cajicá.

Alcaldía Municipal de Fusagasugá (2001): Plan de Ordenamiento Territorial. Fusagasugá.

Alcaldía Municipal de Soacha. Documento técnico de soporte -valoración y formulacióndel Plan de Ordenamiento Territorial. Soacha.

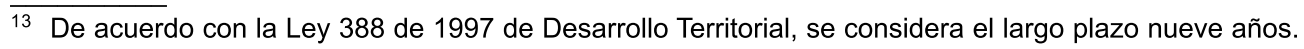

54 Óscar Buitrago B. - Nohora I. Carvajal S. 
Alfonso RoA, Óscar (2002): "Bogotá y la Sabana: crecimiento e institucionalización del ordenamiento en el ámbito de la metropolización. Particularidades del proceso metropolitano de urbanización Bogotá-Sabana: modalidades, implicaciones y determinantes. En: Rentaría, Patricia y Óscar Alfonso: La ciudad: transformaciones, retos y posibilidades. Bogotá: CEJA, Centro Editorial Javeriano.

(Ed.) (2001): Ciudad y región en Colombia. Nueve ensayos de análisis socioeconómico y espacial. Bogotá: Universidad Externado de Colombia.

Buitrago, Óscar (2001): Factores determinantes de los precios de la tierra rural en los municipios contiguos a Bogotá. Tesis de grado para optar al título de magíster en Geografía, Convenio UPTC-IGAC. Bogotá.

CARVAJAL, Nohora y Óscar Buitrago (2003): Informe final del proyecto de investigación "Evaluación de la dimensión urbano regional en los planes de ordenamiento territorial del área metropolitana de Bogota”. Bogotá: Convenio UPTC-IGAC.

Centro Interdisciplinario de Estudios Regionales -CIDER- Universidad de los Andes (2000): Directrices de ordenamiento territorial para Bogotá y la región.

Contraloría de Bogotá D. C. (2003): De Bogotá a la región, apuntes para un modelo de desarrollo regional. Juan Antonio Nieto Escalante (Dirección General). Bogotá. Octubre.

Convenio Corporación Autónoma Regional de Cundinamarca (CAR) - Sociedad Geográfica de Colombia (SGC) (1996): Pre-plan integral físico y ambiental de la cuenca alta del río Bogotá. Bogotá.

Cuervo, Luis Mauricio y Josefina González (1998): Industria y ciudades en la era de la mundialización.

Dureau, Francoise (2002): "Bogotá: una doble dinámica de expansión espacial y de densificación de espacios ya urbanizados". En: Dureau, Francoise et al.: Metrópolis en movimiento. Una comparación internacional. Colombia: Alfaomega.

Gobernación de Cundinamarca. Departamento Administrativo de Planeación de Cundinamarca (1999): Directrices y orientaciones para el ordenamiento territorial del Departamento de Cundinamarca. Bogotá.

GoUËSET, Vincent (1998): Bogotá: nacimiento de una metrópoli. La originalidad del proceso de concentración urbana en Colombia en el siglo Xx. Bogotá: Tercer Mundo Editores.

JARAMILLO, Samuel (1994): Hacia una teoría de la renta del suelo urbano. Bogotá: Ediciones Uniandes - Instituto Geográfico Agustín Codazzi.

MASSIRIS CABEZA, Ángel (1997): “Ordenamiento territorial, región y procesos de construcción regional”. Revista Perspectiva Geográfica, Núm. 1. Tunja: Programa de Estudios de Posgrado en Geografía. 
Mendoza, Alberto (1999): "Bogotá Megalópolis, Metrópolis, Agrópolis. El pacto ecológico". En: Corporación Autónoma Regional de Cundinamarca: Hacia la metropolización de la Sabana de Bogotá. Bogotá

Ministerio de Transporte. Instituto Nacional de Vías. Subdirección de Conservación. Volúmenes de Tránsito 1999.

Montañez, Gustavo (1998): "Universo y parroquialismo en la gestión metropolitana de la Sabana de Bogotá”. En: Misión Local, Núm.10., Bogotá: Universidad Distrital Francisco José de Caldas.

Municipio de BojacÁ. Esquema de Ordenamiento Territorial. Bojacá.

Municipio de Chía (2000): Acuerdo Núm. 17 del 2000 por el cual se adopta el Plan de Ordenamiento Territorial. Chía, junio de 2000.

Municipio del Rosal (1998): Acuerdo que adopta el esquema de ordenamiento territorial municipal. Decreto 789/98. El Rosal.

Municipio de Subachoque (2000): Proyecto de Acuerdo por el cual se adopta el esquema de ordenamiento territorial municipal, se clasifican y determinan usos del suelo y se establecen los sistemas estructurales y planes parciales. Subachoque.

Municipio de ZipaquiRá (1999): Proyecto de acuerdo del plan de ordenamiento territorial. Zipaquirá.

SARMiento, Libardo y Diana Constanza Castillo (1998):. "Regiones y divisiones territoriales en la historia de Colombia". En: Municipios y regiones de Colombia. Fundación Social.

Fecha de recepción: abril de 2004

Fecha de aprobación: agosto de 2004

\begin{tabular}{l|l}
\hline 56 & Óscar Buitrago B. - Nohora I. Carvajal S.
\end{tabular} 